\title{
Acute Kidney Injury in the 2019 Novel Coronavirus Disease
}

\author{
Jing-Yi Qian Bin Wang Bi-Cheng Liu \\ Institute of Nephrology, Zhong Da Hospital, Southeast University School of Medicine, Nanjing, China
}

\section{Keywords}

2019 novel coronavirus disease · COVID-19 · Acute kidney injury

\begin{abstract}
Background: The 2019 novel coronavirus disease (COVID-19) is a newly defined serious infectious disease caused by the SARS-CoV-2 virus. The epidemic started in Wuhan, China, in December of 2019 and quickly spread to over 200 countries. It has affected 4,258,666 people, with 294,190 deaths worldwide by May 15, 2020. COVID-19 is characterized by acute respiratory disease, with $80 \%$ of patients presenting mild like flu-like symptoms; however, $20 \%$ of patients may have a severe or critical clinical presentation, which likely causes multiple organ injuries (e.g., kidney, heart, blood, and nervous system). Among them, acute kidney injury (AKI) is a critical complication due to its high incidence and mortality rate. Here we present a review of the current understanding of AKI in COVID-19. Summary: COVID-19 is a catastrophic contagious disease caused by the coronavirus, and the AKI induced by COVID-19 significantly increases the mortality rate. In this review, we summarize the clinical characteristics of COVID-19 induced AKI by focusing
\end{abstract}

on its epidemiology, pathogenesis, clinical diagnosis, and treatment. Key Messages: Multiple studies have shown that COVID-19 may involve the kidneys and cause AKI. This article reviews the characteristics of COVID-19-induced AKI largely based on up-to-date studies in the hope that it will be helpful in the current global fight against and treatment of COVID-19.

(c) 2020 The Author(s) Published by S. Karger AG, Basel

\section{Introduction}

Novel coronavirus disease (COVID-19) is a newly discovered acute infectious disease caused by the SARS$\mathrm{CoV}-2$ virus, which is mainly manifested as acute respiratory diseases characterized by acute interstitial and alveolar pneumonia and can affect multiple organs such as the kidneys, the heart, the digestive tract, and blood [1]. This disease is highly contagious, with diverse manifestations and complications, and poses a threat to public health, just like another 2 coronavirus-induced diseases, i.e., severe acute respiratory syndrome (SARS) and Middle East respiratory syndrome (MERS). Although it mainly presents with acute pneumonia, acute kidney injury (AKI) is

\begin{tabular}{ll}
\hline karger@karger.com & (c) 2020 The Author(s) \\
Published by S. Karger AG, Basel & Karger \\
www.karger.com/kdd & This article is licensed under the Creative Commons Attribution- \\
NonCommercial-NoDerivatives 4.0 International License (CC BY- \\
NC-ND) (http://www.karger.com/Services/OpenAccessLicense). \\
Usage and distribution for commercial purposes as well as any dis- \\
tribution of modified material requires written permission.
\end{tabular}

Bi-Cheng Liu

Institute of Nephrology, Zhong Da Hospital, Southeast University School of Medicine Dingjiaqiao Rd. 87

Nanjing, Jiangsu 210009 (China)

liubc64@163.com 
definitely not uncommon and plays an important role in the prognosis of severely affected patients. Therefore, much attention must be paid to the AKI in COVID-19.

\section{Epidemiology}

The current outbreak of COVID-19 started in Wuhan, China, in early December of 2019, and it has now spread to over 200 countries [2]. There are 84,464 cumulative confirmed cases, with 4,644 dead in China and 4,174,202 cases with 289,546 dead outside of China by May 15, 2020. In total, about $16-20 \%$ of patients present severe or critical cases. The exact incidence of AKI in COVID-19 is still not fully clear. In the first study reported by Huang et al. [3], the incidence of AKI was 7\%, and 3 out of 13 (23\%) patients in the intensive care unit experienced AKI. In another study of 99 patients with COVID-19, seven cases developed various degrees of kidney injury with elevated serum creatinine (Scr) and/or blood urea nitrogen (BUN) levels, and 3 of them were diagnosed with AKI [4]. Wang et al. [1] reported that $3.6 \%$ of patients developed AKI and 2 patients received renal replacement treatment in his 138-patient cohort study. In a larger multicenter study with 1,019 cases, Guan and Zhong [5] showed that the AKI incidence rate was only $0.5 \%$. However, in a single-center study with 710 consecutive hospitalized COVID-19 patients, Cheng et al. [6] reported that the incidence rate of AKI was 3.2\%; most strikingly, $50 \%$ of the patients with AKI died. Besides, patients with elevated baseline Scr levels tend to develop more severe AKI and have a higher death rate [6]. So it appears that the overall incidence of AKI in COVID-19 is probably lower than that in SARS and MERS $[7,8]$. However, a recent study from Yang et al. [9] suggests that the AKI incidence is quite high $(15 / 52 ; 29 \%)$ in critically ill patients. Quite similar results were also reported by Diao et al. [10] in their retrospective study, demonstrating that $27.06 \%$ $(23 / 85)$ of patients experienced AKI, and elderly patients (age $\geq 60$ years) had a much higher incidence $(69.57 \%$ ) of AKI development [10]. Clearly, AKI in COVID-19 is very common, particularly in patients in severe or critical condition.

\section{Pathogenesis}

Our current understanding of the pathogenesis of AKI in COVID-19 is largely assumptive, based on previous studies and 2 important coronavirus infection, i.e., SARS and MERS. Renal impairment could be directly attacked by the coronavirus or a cytokine storm due to abnormal immunity. Hypotension or dehydration, hypoxemia, sepsis, and nephrotoxic drugs could also be involved in the development of AKI.

\section{Role of Virus Attack}

Angiotensin converting enzyme 2 (ACE2), a metallopeptidase, has been confirmed to effectively bind to the S1 domain of the spike protein on SARS-CoV. Therefore, ACE2 is considered to be a functional receptor of SARS$\mathrm{CoV}$ [11]. Researchers described the full-length genome sequence of SARS-CoV-2 from 5 patients and found that $79.5 \%$ of SARS-CoV-2 sequences are homologous to SARS -CoV [12]. Moreover, they share the same functional receptor, i.e., ACE2, and have an affinity comparable that of human ACE2 (hACE2) [12, 13]. Recently the specific structure of ACE2 was discovered by cryoelectronic microscopy and the multiple conformational states of the SARS-CoV-2 S glycoprotein were demonstrated at a 3.0 - $\AA$ resolution $[13,14]$. hACE2 is not only expressed in lung tissue but it can also be detected in the kidneys, mainly in proximal tubules, afferent arterioles, collecting ducts, and the thick ascending limb of Helen [15]. In addition, viral nucleic acid could also be found in urine in 4 out of 58 (6.9\%), suggesting that the kidneys might be the target of this novel coronavirus [16]. Recently, Diao et al. [10] demonstrated that SARS-CoV-2 mainly induced acute tubular necrosis by infecting kidney tubules directly. Immunohistochemistry demonstrated that the NP antigen of SARS-CoV-2 was accumulated in the cytoplasm of kidney tubules instead of glomeruli based on the autopsy findings of 6 COVID-19 subjects with renal function impairment prior to death [10]. Besides, Su et al. [17] found the virus particles in the cytoplasm of renal proximal tubular epithelium and podocytes but less so in distal tubules. SARS-CoV nucleoprotein was analyzed in 6 cases, and 3 showed positive granular staining in a nuclear or cytoplasm pattern in tubular epithelium. These results provide direct evidence that the SARS-CoV-2 virus can directly infect the renal tubular epithelium and podocytes, which may induce AKI in COVID-19 patients [17]. Thus, the novel coronavirus can induce kidney injury directly via hACE2 or other receptors like SARS-CoV, but the exact mechanism still needs to be clarified.

\section{Role of immune Activation}

Immune activation accompanying the release of a large amount of proinflammatory factors may also play an important role in COVID-19-associated AKI. Previ- 
ous studies have demonstrated that SARS-CoV and MERS-Cov infection could lead to a significant upregulation of inflammatory factors and chemokines [18]. It is noteworthy that cytokine storm syndrome (CSS) may play a critical role in multiple organ dysfunction syndrome in severe patients. Laboratory examination showed that patients with COVID-19 have a higher level of TNF- $\alpha$, IL-1, IL-6, interleukin (IL)-12, and interferon (IFN)- $\alpha$, suggesting that they may have experienced CSS. Chen et al. Recently a significant upregulation of IL- 6 and the IL-2 receptor (IL-2R) was demonstrated in 29 patients with COVID-19 [19]. The exact role of CSS in COVID-19 needs to be further investigated.

\section{Role of Other Clinical Factors}

Severely or critically illness patients with COVID-19 often experience hypotension, hypoxemia, diarrhea, dehydration, electrolyte and acid-base balance disorder, cardiac insufficiency, and disseminated intravascular coagulation. Meanwhile, drugs like NSAID, antiviral drugs, antibiotics, and (or) norepinephrine could all be attributable to the development of AKI, specifically in aging patients with underlying diseases like diabetes, cardiovascular disease, or cancer.

\section{Clinical Manifestations of AKI in COVID-19}

\section{General Manifestation}

COVID-19 can affect a variety of organs and systems, including respiratory, urinary, digestive, cardiovascular, blood, and neurological systems, depending on the severity [1]. The most common symptoms at the onset of the COVID-19 illness are fever, cough, and fatigue, while other symptoms include sputum production, headache, hemoptysis, diarrhea, dyspnea, and lymphopenia. Severe patients may experience a high fever, anhelation, chest distress, cyanosis, hypoxemia, hypotension, etc. The main feature of lung injury is acute interstitial pneumonia, i.e., alveolar pneumonia with a large amount mucinous secretion, which usually causes persistent hypoxemia. There are other relatively rare complications like acute respiratory distress syndrome and acute cardiac injury $[2,3,20]$.

\section{Renal Manifestations}

Patients with COVID-19 may have various degree of renal dysfunction, characterized by elevation of BUN, creatinine $(\mathrm{Cr})$, and renal structural changes [4]. A study of 59 patients with COVID-19 found that $34 \%$ of patients developed massive albuminuria on the first day of admission and $63 \%$ of patients presented proteinuria during their stay in hospital [21]. BUN was elevated in $27 \%$ of the patients, and more importantly two thirds of the patients who died presented with increased BUN and Scr over 200 $\mu \mathrm{mol} / \mathrm{L}$. Kidney CT scans for showed the density was in the range of 19.5-34.97 HU, which is significantly lower than value in patients without kidney disease (i.e., $35 \mathrm{HU}$ ). This result indicates that inflammation and edema of the renal parenchyma may occur in patients with COVID-19 [21]. Cheng et al. [6] recently reported that $44 \%$ of patients presented with proteinuria and hematuria and $26.9 \%$ had hematuria on admission among 710 consecutive hospitalized patients with COVID-19, and the prevalence of elevated Scr and BUN levels was 15.5 and $14.1 \%$, respectively. Although the incidence of AKI was 3.2\%, it is an independent risk factor for in-hospital death [6]. Recent autopsy data demonstrated that tubular epithelial cell necrosis and degeneration, with interstitial hyperemia, microthrombus, or focal fibrosis were the main pathological features, while glomerular lesions are not common [2].

\section{Laboratory Examination}

In the early stage of the disease, the number of peripheral blood leukocytes is normal or reduced; the lymphocyte count is reduced as well. Some patients may suffer from liver disorder with abnormally elevated alanine aminotransferase, aspartate aminotransferase, and lactate dehydrogenase levels. Elevated troponin can be seen in some critically ill patients. C-reactive protein and erythrocyte sedimentation rates increased in most patients, and procalcitonin was usually normal. In severe cases, D-dimer increased and peripheral blood lymphocytes decreased progressively [2]. Chest CT showed multiple small patch shadows and interstitial changes in the early stage, which further developed into multiple ground glass shadows and infiltration shadows in both lungs. In severe cases, consolidation of the lung or white lung may occur, while pleural effusion is rare [2]. Moreover, a study of 52 critically ill patients showed that lymphocytopenia occurred in more than $80 \%$ of COVID-19 patients [7]. However, a previous study claimed that $35 \%$ of noncritical patients infected with SARS-CoV-2 had mild lymphocytopenia [4], suggesting that the severity of lymphocytopenia reflects the severity of SARS-CoV-2 infection. A recently published case report analyzed the kinetics of immune responses of a nonsevere case of COVID-19, which suggest that immune parameters should be tested in COVID-19. Early effective adaptive immune responses might correlate with better clinical outcomes [22]. 


\section{Diagnosis}

The diagnosis of AKI complicated with COVID-19 is based on the diagnosis of COVID-19 and complied with the criteria of KDIGO [1]. AKI is identified as one of the following: (1) an Scr increase $\geq 0.3 \mathrm{mg} / \mathrm{dL}(\geq 26.5 \mu \mathrm{mol} / \mathrm{L})$ within $48 \mathrm{~h},(2)$ an Scr increase to $\geq 1.5$ times baseline within the previous 7 days; or (3) a urine volume $\leq 0.5$ $\mathrm{mL} / \mathrm{kg} / \mathrm{h}$ for $6 \mathrm{~h} \mathrm{[23].}$

For diagnosis of COVID-19, epidemiological factors, clinical manifestations, and laboratory examination (including a hemogram, a chest $\mathrm{CT}$, a virological examination, etc.) should be combined [2]. Novel coronavirus nucleic acid detection, gene sequencing, or specific antibodies (both IgM and IgG) are all recommended approaches for confirmed diagnosis [2]. However, false negativity may happen in some cases with a gene detection kit; it should therefore be used carefully and repeat analysis or combined use with other approaches is recommended [2]. Currently, detection kits like Reverse Transcription Loop-Mediated Isothermal Amplification (RT-LAMP) with better sensitivity and specificity are under investigation [24].

\section{Treatment}

The main treatment of COVID-19 with AKI includes general management, antiviral therapy, renal replacement therapy, and other supportive therapies.

\section{General Management}

All patients with confirmed COVID-19 should be placed in effective quarantine and treated in designated hospitals. Moreover, early admission to an intensive care unit (ICU) is recommended for critical patients. Patients are given rest, supportive treatment such as nutrients, and maintenance of homeostasis. Oxygen therapy should be provided when necessary. Fever is a common symptom of COVID-19, and nonsteroidal anti-inflammatory drugs (NSAID) are given cautiously. The management of critical cases should focus on the prevention and treatment of complications by maintaining hemodynamic stability. In addition, prevention of the secondary infection should also be emphasized. Timely administration of high-flow oxygen, mechanical ventilation, or extracorporeal membrane oxygenation are key approaches for those with ARDS. It should be noted that assessing the patient's psychological situation and relieving the anxiety of patients are also integral parts of coping with this disorder [2].

\section{Antiviral Therapy}

Although antiviral therapy is critical, there is currently no confirmed effective antiviral drug for COVID-19. According to the updated version of guidelines recommended by the National Health Commission of the People's Republic of China, aerosol inhalation of IFN- $\alpha$ and lopinavir/ritonavir are suggested for patients [2]. The specific therapeutic value and safety of lopinavir/ritonavir in COVID-19 patients are under investigation (ChiCTR2000029308). The efficacy of the nucleoside analog GS-5734 (remdesivir) has been confirmed both in SARS$\mathrm{CoV}$ and in MERS-CoV [25, 26]. These studies suggested that remdesivir might be considered for a wider range of coronaviruses, including the current COVID-19. Successful treatment with remdesivir has been reported in COVID-19 patients [27], which quickly piqued the interest of Chinese doctors. TA strictly designed clinical trial on the efficacy of remdesivir in COVID-19 patients was started in China on February 3 (NCT04252664 and NCT04257656) and will hopefully be finished in April of 2020.

\section{Blood Purification}

Blood purification comprises a group of techniques including plasma exchange, adsorption, perfusion, and continuous renal replacement therapy (CRRT). Among them, CRRT is critical for severe COVID-19 cases due to its unique technical features, specifically for those with AKI, systemic inflammatory response syndrome, multiple organ dysfunction syndrome, and CSS. CRRT is usually recommended for those with hyperkalemia, acidosis, pulmonary edema, or water overloaded [2]. Besides, it should be noted that CRRT may play a crucial role in clearance of overloaded inflammatory cytokines in severe and critical patients [28]. Ghani et al. [29] had showed the efficacy of high-volume hemofiltration $(6 \mathrm{~L} / \mathrm{h})$ treatment in sepsis, with not only removed inflammatory cytokines (IL-6, $p=0.025$ ) but also improved SOFA (Sequential Organ Failure Assessment) scores on day 7. Park JT et al. [30] also demonstrated that a high dose of CRRT could significantly reduce the patient's serum IL-6 and IL-8 levels. In a randomized clinical trial, Zarbock et al. [31] demonstrated that early use of RRT (KDIGO Stage II started in less than $8 \mathrm{~h}$ ) could significantly reduce the mortality rate in 90 days compared to delayed use (KDIGO stage III started in over $12 \mathrm{~h}$ ) in critically ill patients [31]. All of these studies suggested that timely use CRRT might be an effective therapy in helping to clear the deadly cytokine storm in severe patients of COVID-19. 
Other Therapies

Convalescent Plasma

Preliminary clinical studies in China have showed that early application of convalescent plasma in patients with COVID-19 is an effective approach and could accelerate the patients' clinical recovery [32]. However, van Griensven et al. [33] reported that transfusion of up to $500 \mathrm{ml}$ of convalescent plasma with unknown levels of neutralizing antibodies to 84 patients with confirmed Ebola virus disease did not show significant improvement in survival. The reason for this discrepancy is still unclear. Now 2 trials (an open-label, non-randomized clinical trial [NCT04264858] and a multicenter, randomized, and parallel controlled trial [ChiCTR2000029757]) about the efficacy of convalescent plasma in patients with COVID-19 are ongoing in China.

Monoclonal antibody directed against the RBD domain of the $S$ protein of MERS-CoV has been verified to be effective in vitro [34]. Although the monoclonal antibody against COVID-19 has not been developed, trastuzumab, as a monoclonal antibody against IL-6 receptor, achieved encouraging results in a preliminary clinical observation. The safety and effectiveness evaluation of trastuzumab is ongoing in multicenter randomized controlled trials (ChiCTR2000029765).

\section{Glucocorticoids}

Glucocorticoids are usually applied in severely ill patients with a viral infection. In the retrospective study im SARS-CoV, it was found that steroids could reduce the mortality and shorten the hospitalization time [35]. However, the use of steroids is still quite controversial due to its potential inhibition of virus clearance and prolongation of the duration of viremia.

\section{Conclusion}

SARS, MERS, and COVID-19, as the 3 catastrophic contagious diseases caused by coronavirus in this century, pose great threats to public health and the safety of the world. AKI as a complication of COVID-19 could not only significantly increase the mortality rate of COVID-19 but also, in the long run, increase the risk of developing chronic kidney disease. It is therefore important to pay more attention to finding $\mathrm{AKI}$ in a timely manner and treat the patients properly when we are fighting COVID-19.

\section{Acknowledgement}

We would like to thank all of the medical staff at the Institute of Nephrology of Zhong Da Hospital for their support in writing this article.

\section{Conflict of Interest Statement}

The authors have no conflict of interests to disclose, and the results in this paper have not been published previously in whole or part.

\section{Funding Sources}

Financially support for this work was provided by the key international cooperation program of the China National Natural Science Foundation (81720108007) and the key research project of the Ministry of China Science and Technology (2018YFC1314000) to Prof. Bi-Cheng Liu as PI.

\section{Author Contributions}

Bi-Cheng Liu designed this study, Jing-Yi Qian carried out this study, Jing-Yi Qian and Bin Wang wrote the draft, and Bi-Cheng Liu revised this paper.

\section{References}

1 Wang D, Hu B, Hu C, Zhu F, Liu X, Zhang J, et al. Clinical Characteristics of 138 Hospitalized Patients With 2019 Novel Coronavirus-Infected Pneumonia in Wuhan, China. JAMA. 2020 Feb;323(11):1061.

2 National Health Commission of the People's Republic of China. Guidelines for diagnosis and treatment of 2019 novel coronavirus disease (version 7). Beijing: National Health Commision of the People's Republic of China;2020.

3 Huang C, Wang Y, Li X, Ren L, Zhao J, Hu Y, et al. Clinical features of patients infected with 2019 novel coronavirus in Wuhan, China. Lancet. 2020 Feb;395(10223):497-506.

4 Chen N, Zhou M, Dong X, Qu J, Gong F, Han $\mathrm{Y}$, et al. Epidemiological and clinical charac- teristics of 99 cases of 2019 novel coronavirus pneumonia in Wuhan, China: a descriptive study. Lancet. 2020 Feb;395(10223):507-13.

5 Guan WJ, Zhong NS. Clinical Characteristics of Covid-19 in China. Reply [Reply]. N Engl J Med. 2020 May;382(19):1861-2.

6 Cheng Y, Luo R, Wang K, Zhang M, Wang Z, Dong $\mathrm{L}$, et al. Kidney disease is associated with in-hospital death of patients with COVID-19 [published online ahead of print, 2020 Mar 20]. Kidney Int. 2020 May;97(5):829-38.

7 Eckerle I, Müller MA, Kallies S, Gotthardt DN, Drosten C. In-vitro renal epithelial cell infection reveals a viral kidney tropism as a potential mechanism for acute renal failure during Middle East Respiratory Syndrome
(MERS) Coronavirus infection. Virol J. 2013 Dec;10(1):359-63.

8 Chu KH, Tsang WK, Tang CS, Lam MF, Lai FM, To KF, et al. Acute renal impairment in coronavirus-associated severe acute respiratory syndrome. Kidney Int. 2005 Feb;67(2): 698-705.

9 Yang X, Yu Y, Xu J, Shu H, Xia J, Liu H, et al. Clinical course and outcomes of critically ill patients with SARS-CoV-2 pneumonia in Wuhan, China: a single-centered, retrospective, observational study [published online ahead of print, 2020 Feb 24] [published correction appears in Lancet Respir Med. 2020 Feb 28]. Lancet Respir Med. 2020 May;8(5): 475-81. 
10 Diao B, Wang CH, Wang RS, Feng ZQ, Tan YJ, Wang HM, et al. Human kidney is a target for novel severe acute respiratory syndrome coronavirus 2 (SARS-CoV-2) infection. medRxiv. 2020. Available from: https://doi.org/ https://doi.org/10.1101/2020.03.04.20031120.

11 Li W, Moore MJ, Vasilieva N, Sui J, Wong SK, Berne MA, et al. Angiotensin-converting enzyme 2 is a functional receptor for the SARS coronavirus. Nature. 2003 Nov;426(6965): $450-4$.

12 Zhou P, Yang XL, Wang XG, Hu B, Zhang L, Zhang $\mathrm{W}$, et al. A pneumonia outbreak associated with a new coronavirus of probable bat origin. Nature. 2020 Mar;579(7798):270-3.

13 Walls AC, Park YJ, Tortorici MA, Wall A, McGuire AT, Veesler D. Structure, Function, and Antigenicity of the SARS-CoV-2 Spike Glycoprotein. Cell. 2020 Apr;181(2):281292.e6.

14 Yan R, Zhang Y, Li Y, Xia L, Guo Y, Zhou Q. Structural basis for the recognition of SARSCoV-2 by full-length human ACE2. Science. 2020 Mar;367(6485):1444-8.

15 Santos RA, Ferreira AJ, Verano-Braga T, Bader $\mathrm{M}$. Angiotensin-converting enzyme 2, angiotensin-(1-7) and Mas: new players of the renin-angiotensin system. J Endocrinol. 2013 Jan;216(2):R1-17.

16 Ling Y, Xu SB, Lin YX, Tian D, Zhu ZQ, Dai $\mathrm{FH}$, et al. Persistence and clearance of viral RNA in 2019 novel coronavirus disease rehabilitation patients. Chin Med J (Engl). 2020 May;133(9):1039-43.

17 Su H, Yang M, Wan C, Yi LX, Tang F, Zhu $\mathrm{HY}$, et al. Renal histopathological analysis of 26 postmorten findings of patients with COVID-19 in China. Kidney Int. 2020. doi: 10.1016/j.kint.2020.04.003.

18 Arabi YM, Balkhy HH, Hayden FG, Bouchama A, Luke T, Baillie JK, et al. Middle East Respiratory Syndrome. N Engl J Med. 2017 Feb;376(6):584-94.

19 Chen L, Liu HG, Liu W, Liu J, Liu K, Shang J, et al. Analysis of clinical features of 29 patients with 2019 novel coronavirus pneumonia. Zhonghua Jie He He Hu Xi Za Zhi. 2020 Mar; 43(3):203-8.
20 Rothan HA, Byrareddy SN. The epidemiology and pathogenesis of coronavirus disease (COVID-19) outbreak. J Autoimmun. 2020 May; 109:102433.

21 Anti-2019-nCoV volunteers; $\mathrm{Li} Z$ Z, Wu M, Yao JW, Guo J, Liao X, et al. Caution on kidney dysfunctions of 2019-nCoV patients. medRxiv. 2020. Available from: https://doi.org/https:// doi.org/10.1101/2020.02.08.20021212.

22 Thevarajan I, Nguyen TH, Koutsakos M, Druce J, Caly L, van de Sandt CE, et al. Breadth of concomitant immune responses prior to patient recovery: a case report of non-severe COVID-19. Nat Med. 2020 Apr;26(4):453-5.

23 Kidney Disease: Improving Global Outcomes (KDIGO) Acute Kidney Injury Work Group. KDIGO clinical practice guideline for acute kidney injury. Kidney Int Suppl. 2012;2:1.

24 Lamb LE, Bartolone SN, Ward E, Chancellor MB. Rapid detection of novel coronavirus (COVID-19) by reverse transcription-loopmediated-isothermal amplification. medRxiv. 2020. Available from: https://doi.org/https:// doi.org/10.1101/2020.02.19.20025155.

25 Agostini ML, Andres EL, Sims AC, Graham RL, Sheahan TP, Lu X, et al. Coronavirus Susceptibility to the Antiviral Remdesivir (GS5734) Is Mediated by the Viral Polymerase and the Proofreading Exoribonuclease. MBio. 2018 Mar;9(2):e00221-18.

26 de Wit E, Feldmann F, Cronin J, Jordan R, Okumura A, Thomas T, et al. Prophylactic and therapeutic remdesivir (GS-5734) treatment in the rhesus macaque model of MERSCoV infection. Proc Natl Acad Sci USA. 2020 Mar;117(12):6771-6.

27 Holshue ML, DeBolt C, Lindquist S, Lofy KH, Wiesman J, Bruce H, et al.; Washington State 2019-nCoV Case Investigation Team. First case of 2019 novel coronavirus in the United States. N Engl J Med. 2020 Mar;382(10):92936.
28 Zhou Y, Zhou F, Wang X, Chang P, Zhang L, Yao Q, et al. Practice of Extracorporeal Therapies for Septic Acute Kidney Injury Patients in Intensive Care Units in Mainland China. Blood Purif. 2019;47 Suppl 3:1-6.

29 Ghani RA, Zainudin S, Ctkong N, Rahman AF, Wafa SR, Mohamad M, et al. Serum IL-6 and IL-1-ra with sequential organ failure assessment scores in septic patients receiving high-volume haemofiltration and continuous venovenous haemofiltration. Nephrology (Carlton). 2006 Oct;11(5):386-93.

30 Park JT, Lee H, Kee YK, Park S, Oh HJ, Han $\mathrm{SH}$, et al.; HICORES Investigators. High-dose versus conventional-dose continuous venovenous hemodiafiltration and patient and kidney survival and cytokine removal in sepsis-associated acute kidney injury: a randomized controlled trial. Am J Kidney Dis. 2016 Oct;68(4):599-608.

31 Zarbock A, Kellum JA, Schmidt C, Van Aken $\mathrm{H}$, Wempe C, Pavenstädt $\mathrm{H}$, et al. Effect of early vs delayed initiation of renal replacement therapy on mortality in critically ill patients with acute kidney injury-the ELAIN randomized clinical trial. JAMA. 2016 May; 315(20):2190-9.

32 Joint defense and control mechanism of the State Council. Press conference of the joint defense and control mechanism of the State Council. 2020 Feb 17.

33 van Griensven J, Edwards T, de Lamballerie X, Semple MG, Gallian P, Baize S, et al.; Ebola-Tx Consortium. Evaluation of convalescent plasma for Ebola virus disease in Guinea. N Engl J Med. 2016 Jan;374(1):33-42.

34 Park BK, Maharjan S, Lee SI, Kim J, Bae JY, Park MS, et al. Generation and characterization of a monoclonal antibody against MERS$\mathrm{CoV}$ targeting the spike protein using a synthetic peptide epitope-CpG-DNA-liposome complex. BMB Rep. 2019 Jun;52(6):397-402.

35 Chen RC, Tang XP, Tan SY, Liang BL, Wan ZY, Fang JQ, et al. Treatment of severe acute respiratory syndrome with glucosteroids: the Guangzhou experience. Chest. 2006 Jun; 129(6):1441-52. 